\title{
Exploring women's perceptions and experiences of menopause among East Coast Malaysian women
}

Nurul Najmi Mohamad Ishak, Nurjasmine Aida Jamani, Siti Roshaidai Mohd Arifin, Azwanis Abdul Hadi, Karimah Hanim Abd Aziz

Mohamad Ishak NN, Jamani NA, Mohd Arifin SR, et al. Exploring Women's Perceptions and Experiences of menopause among East Coast

Malaysian Women. Malays Fam Physician. 2021;16(1);84-92. https://doi.org/10.51866/oa1098

\section{Keywords:}

Menopause, experience,

Malaysia, women

\section{Authors:}

Nurjasmine Aida Jamani

(Corresponding author)

MD, M. Med (Fam Med), IBCLC

Department of Family Medicine

Kulliyyah of Medicine, International

Islamic University Malaysia

Pahang, Malaysia

email: minaida@iium.edu.my

Nurul Najmi Mohamad Ishak

MBBS

Department of Family Medicine

Kulliyyah of Medicine, International

Islamic University Malaysia

Pahang, Malaysia

Siti Roshaidai Mohd Arifin

$\mathrm{PhD}$ (Midwifery), MNSc,

BHSc, RN, RM

Department of Special Care Nursing Kulliyyah of Nursing International Islamic University Malaysia

Pahang, Malaysia

\section{Azwanis Abdul Hadi}

MBChB, M.Med (Fam Med)

Deparment of Family Medicine

Kulliyyah of Medicine, International

Islamic University Malaysia

Pahang, Malaysia

\section{Abstract}

Background: Menopause signifies the beginning of a non-reproductive phase from the reproductive period. Although it is considered a natural life transition, recent studies have indicated that a woman's menopausal experience may impact her quality of life. While studies have examined Malaysian women's knowledge and awareness of menopause, they have focused on the prevalence of menopausal symptoms and sociocultural aspects. These investigations also used a quantitative design that may have limited the information regarding the nature and experience of menopause.

Objectives: This study explores women's perceptions and understanding of their menopausal experience.

Methods: A total of 20 menopausal women were recruited using purposive sampling through social media in the state of Pahang, Malaysia. In-depth semi-structured interviews were used to explore how they perceived their menopausal experience. The data were then analyzed using thematic analysis.

Results: Three themes that emerged from the findings included perceptions of menopause, biopsychosocial changes and help-seeking behavior. For many Muslim women, menopause was perceived as a time for them to engage in religious commitment more than they had before.

Conclusion: The study findings uncovered a knowledge gap and a lack of support for women impacted by menopause. Muslim women regarded their menopause phase as a time for them to engage in religious duty fully. These results indicate the need for a psychosocial intervention and educational program to ensure women a better transition throughout their menopausal phase.

\section{Introduction}

Menopause comes from the Greek word "meno," meaning month, and "pausia," which means stop or halt. ${ }^{1}$ Menopause signifies the end of menstruation due to the loss of ovarian follicular activity resulting in reduced estrogen and progesterone levels. ${ }^{2}$ This phenomenon marks the beginning of a non-reproductive phase following the reproductive phase. During this phase, women might face a struggle in coping with menopausal symptoms. Symptoms such as hot flushes, fatigue, joint and muscle pain, headache, urinary problems, depression, and mood swings are commonly reported.

While menopause is considered a natural life transition, recent studies have shown that women's menopausal experience may impact their quality of life.,4 For example, some women have experienced negative mood and depressive symptoms during the menopausal transition. ${ }^{5}$ Moreover, researchers have reported that common menopausal symptoms such as sleep disturbance and cognitive problems negatively impacted women's work and relationship. ${ }^{6}$

Previous studies have indicated that menopausal women preferred to seek help from informal sources, such as friends and relatives, rather than healthcare providers, suggesting that women perceive menopause as a natural event. ${ }^{4,7}$ Evidence revealed that a lack of knowledge regarding treatment available for menopause resulted in not seeking help from formal sources such as healthcare providers. For example, Alshogran et $\mathrm{al}^{8}{ }^{8}$ found that most of the participants in their study did not know the roles, benefits, and risks of hormone replacement therapy (HRT).

Similarly, other studies reported that most menopausal women were uncertain about how long their menopausal symptoms might last and were not aware of hormone replacement therapy. ${ }^{6,9}$ Although some women revealed a sufficient understanding of the immediate effects of menopause, they exhibited a lack 
Karimah Hanim Abd Aziz $\mathrm{MPH}, \mathrm{DrPH}$

Department of Community Medicine Kulliyyah of Medicine, International Islamic University Malaysia Pahang, Malaysia of knowledge of the long-term consequences and reflected a negative attitude towards therapy. ${ }^{10}$ Furthermore, Stanzel et al.'s review of 19 studies $^{11}$ indicated that self-management strategies for menopausal symptoms were influenced by culture where menopause was considered a normal condition. Inadequate health-related knowledge about and awareness of menopause as evidenced in the above studies resulted in poor treatment-seeking behaviour. ${ }^{9,12}$

Studies found several barriers that hindered menopausal women from seeking help, including low menopause-related health literacy, perceived low priority for seeking help for symptoms, limited availability of health services and cost, financial issues, and considering menopause a part of the normal aging process., ${ }^{4,13}$ Some evidence revealed that many menopausal women appeared to "normalize" their menopausal symptoms or viewed menopause as a natural event and commonly sought menopause-related information from family and friends as a result. $^{4,14}$

While women often felt isolated, they were reluctant to talk openly about menopause or consult a healthcare professional. ${ }^{6}$ Some women felt dissatisfied with the care they had received for menopause and its treatment and considered the information and support available to them insufficient. ${ }^{6,11}$ Perceptions of menopause as a normal life phase also contributed to non-help-seeking behavior in the majority of women across countries and cultures. ${ }^{4,6}$

Although a few studies have evaluated women's knowledge and awareness of menopause in Malaysia, ${ }^{15-18}$ most studies focused on the prevalence of menopausal symptoms and sociocultural aspects. These studies also used a quantitative design that might have limited the information on the nature and experience of menopause. Furthermore, the use of quantitative approaches (e.g., cross-sectional design and self-reported questionnaire) in the majority of these studies limited further explanation of Malaysian women's experience of menopause.

A comprehensive exploration of women's experience of menopause is essential. The information sought could be applied by healthcare providers to evaluate, enhance, and promote future menopause education programs. In particular, this study's findings will form the basis for future interventions for menopausal women in Malaysia.

Thus, this study explores menopausal experience in postmenopausal women, their knowledge and perceptions toward menopause, self-help strategies in managing menopause, and the barriers to seeking help or treatment.

\section{Method}

\section{Theoretical Framework}

Evidence shows that the experience of menopausal symptoms is influenced by individual, cultural, social, and psychological factors. ${ }^{19,20}$ Thus, it seems relevant to apply the self-regulation model to explain women's cognitive representations of menopause and how they cope with their experienced symptoms. ${ }^{21}$

According to this theory, behavior is guided by a motivational system of setting goals, developing and enacting strategies to achieve those goals, evaluating progress, and revising goals and actions accordingly. Self-regulation models are commonly used to explain healthrelated behaviors adopted to improve health, such as avoiding sources of stress, obtaining informal sources of health information, seeking medical care, adhering to prescribed medication, and performing regular physical exercise. $^{22}$

The basic element of the model is the concept of feedback. In the case of menopause, a woman who understands that menopause is a condition requiring certain behavioral changes will search for information about the outcomes of her behaviors and move toward the desired outcome. This model also emphasizes selfcontrol, willpower, and the role of emotions in motivating and guiding behavior.

\section{Study design and setting}

This study is a qualitative research design using purposive sampling involving 20 women who had reached menopause in Kuantan, Pahang. Accordingly, the women selected in this study presented particular characteristics that enable a detailed exploration and understanding of menopause in Malaysia.

Purposive sampling is a vital sampling method in a qualitative study that entails 
identifying and selecting individuals or groups of individuals who are knowledgeable or have experienced a phenomenon of interest based on the selection criteria. ${ }^{23-24}$ In this study, women were purposely selected based on their age and years of menopause.

A qualitative method was used to explore the experience of menopause among these women since this approach offers the advantage of exploring women's experiences comprehensively through in-depth interviews, helping the researcher understand how menopausal women make sense of their lives and their experiences. Furthermore, this practice comprises the most frequent type of qualitative study done in all fields of practice, including the health sector.

In-depth interviews were chosen for this study as a more appropriate investigation tool for disclosing personal, sensitive information such as attitude and experience related to intimate issues compared to focus group discussion. ${ }^{25}$ In this case, menopause is considered a sensitive issue among Asian women; this study aimed to provide insight into how women interpreted their menopausal experience. ${ }^{26}$

\section{Sampling}

The participants were recruited through advertising on social media platforms such as Facebook, WhatsApp, and Instagram. Inclusion criteria and the researchers' contact details were included in the advertisement. The inclusion criteria were women who had experienced natural menopause within 5 years based on their recalled last menstrual period and symptoms of menopause. Women who had surgical menopause, premature menopause, and psychiatric illness were excluded.

Women who fulfilled the criteria and agreed to participate were given a date for the interview, which was determined based on the researchers' and respondents' availability. Data were collected for a period of 4 months, from May 2018 to August 2018.

Sample size is estimated based on the concept of saturation for qualitative research. Based on Dworkin ${ }^{27}$, a minimum of 5 participants and a maximum of 50 participants comprised an adequate sample size for using in-depth interviews. In this study, no new themes emerged after 15 respondents. Nevertheless, the researchers increased the recruitment of respondents up to 20 postmenopausal women to ensure data saturation was reached.

\section{Data collection}

Participants were invited for interviews at a time, date, and location that suited their convenience and preference. The semi-structured in-depth interviews were conducted using open-ended questions. The topic guide was developed based on the research questions that addressed menopausal knowledge, experiences, attitudes, and beliefs about menopause, along with the symptoms and ways of coping with menopause. We prepared an interview guide (Table 1), and the interview questions were established following a literature review. ${ }^{28-31}$ Two pilot interviews were conducted to refine the topic guide and evaluate and enhance the rigor of the interview sessions.

Interviews were conducted in either the Malay language or the English language. For this study, all interviews were conducted in the participants' houses. The participants provided written consent and brief sociodemographic information before the interview sessions. Confidentiality and anonymity were maintained. Each interview session was conducted only once for each participant, and each lasted for 45 to 60 minutes. The data from the interviews were audio-recorded and transcribed verbatim.

\section{Data analysis}

The data from this study were manually analyzed using thematic analysis as described by Braun and Clarke. ${ }^{32}$ During analysis, the transcript was first read to encourage data immersion, after which codes were determined by highlighting words or phrases from the text to capture key concepts.

Notes were made concerning first impressions of the data, and the codes were sorted into categories based on links. These categories organized the codes into meaningful clusters, facilitating deriving themes. The advantage of this analysis method is the ability to gain direct information without imposing preconceived categories. However, it may fail to identify key categories. Once all investigators agreed that the categories were saturated, the recruitment process was finalized. 
Table 1. Topic Guide

\begin{tabular}{|c|c|}
\hline $\begin{array}{l}\text { Women's experience during } \\
\text { menopause }\end{array}$ & Questions \\
\hline $\begin{array}{l}\text { Experiences of women during } \\
\text { menopause }\end{array}$ & $\begin{array}{l}\text { 1. Can you tell me about your life after cessation of menses? } \\
\text { 2. Could you tell me what symptoms have you encountered during } \\
\text { menopause? } \\
\text { 3. Can you tell me how menopause affects your general health? }\end{array}$ \\
\hline $\begin{array}{l}\text { Effect of menopause on } \\
\text { women's quality of life? }\end{array}$ & 1. Can you tell me how menopause affects your life in any way? \\
\hline Coping with the symptoms & $\begin{array}{l}\text { 1. How do you deal/cope with menopausal symptoms? } \\
\text { 2. What kind of assistance have you needed? Why did you choose it? }\end{array}$ \\
\hline Help-seeking & 1. Can you tell me why you didn't see your health practitioner? \\
\hline
\end{tabular}

We used maximum variance among participants where the participants had different levels of education and social background to ensure credibility. Furthermore, a member checking process entailed setting appointments to visit the participants in their homes. The participants read the polished content of the transcripts and the quotations cited. Rich, thick description was used to provide many perspectives about a theme to allow the results to be richer and realistic.

\section{Ethical Approval}

This study was approved by the International Islamic University Malaysia Research Ethics Committee with the project code IREC2018-069. All participants received verbal and written information before signing the informed consent form. All were informed about confidentiality and the right to withdraw their consent to participate in this study. Personal details have been changed to ensure that the participants are not identifiable.

\section{Results}

\section{Demographic characteristics}

In this study, 20 menopausal women between the ages of 49 and 61 years participated voluntarily, as shown in Table 2.

Most participants were Malays, were married, and had 3 or more children. Half of them were employed and had secondary education. Most had their menopause within the previous 3 years.

Table 2. Descriptive characteristics of participants

\begin{tabular}{|c|c|c|c|c|c|c|c|c|}
\hline No & Pseudonym & $\begin{array}{c}\text { Age at } \\
\text { interview }\end{array}$ & Race* & $\begin{array}{c}\text { Marital } \\
\text { status }\end{array}$ & Parity & $\begin{array}{c}\text { Years of } \\
\text { menopause }\end{array}$ & $\begin{array}{c}\text { Education } \\
\text { level }\end{array}$ & Occupation \\
\hline 1 & S & 58 & M & Divorce & 1 & 3 & Primary & Housewife \\
\hline 2 & H & 54 & M & Married & 5 & 1 & Secondary & Therapist \\
\hline 3 & A & 57 & M & Married & 4 & 1 & Tertiary & Staff nurse \\
\hline 4 & WH & 52 & M & Married & 6 & 2 & Secondary & Housewife \\
\hline 5 & RM & 58 & M & Married & 3 & 4 & Secondary & Community nurse \\
\hline 6 & AS & 54 & M & Single & 0 & 1 & Secondary & Food staller \\
\hline 7 & Z & 56 & M & Married & 5 & 3 & Secondary & Frozen food retailer \\
\hline 8 & HS & 59 & M & Married & 4 & 4 & Tertiary & Pensioner \\
\hline 9 & N & 49 & M & Single & 0 & 1 & Tertiary & Consultant \\
\hline 10 & ZD & 54 & M & Divorce & 6 & 4 & Secondary & Cleaner \\
\hline 11 & C & 54 & C & Married & 3 & 4 & Secondary & Housewife \\
\hline 12 & F & 53 & M & Married & 4 & 2 & Primary & Food staller \\
\hline 13 & ZK & 56 & M & Married & 4 & 3 & Primary & Clerk \\
\hline 14 & NG & 53 & M & Divorce & 0 & 2 & Tertiary & Procurement analyst \\
\hline 15 & NS & 55 & M & Married & 2 & 3 & Tertiary & Housewife \\
\hline 16 & RO & 54 & M & Married & 3 & 3 & Tertiary & Business-woman \\
\hline 17 & AM & 58 & M & Divorce & 7 & 5 & Primary & Housewife \\
\hline 18 & NJ & 49 & M & Married & 5 & 2 & Secondary & Housewife \\
\hline 19 & L & 61 & C & Married & 4 & 3 & Secondary & Housewife \\
\hline 20 & PM & 57 & I & Married & 2 & 5 & Secondary & Housewife \\
\hline & & & & & & & & \\
\hline
\end{tabular}

* Note: M-Malay, C-Chinese I- Indian 


\section{Menopausal women's experiences}

Three themes were identified to explain the women's experiences with menopause: perception of menopause, biopsychosocial changes, and help-seeking behavior. The following discussion presents details about the themes and selected quotations from the interviews.

\section{Theme 1: Perception of menopause}

The findings indicated that the participants perceived menopause as a negative element in their lives, indicating the beginning of growing old and a phase involving health deterioration. One of them mentioned:

"Since menopause, I always get sick. I easily get fever, cough and flu. It's almost two years I felt like this. This year, almost every month I need to go to the clinic for being unwell. I fainted last year. I have done my medical checkup, and everything was told to be normal. I don't know why it happened. My general health has not been good since menopause. Before this, I seldom fall sick."

\section{(ZK, 56, Malay)}

However, some women perceived menopause as a positive event, marking the end of the reproductive phase. One woman commented:

"When we have reached menopause, we don't need to think about pregnancy. We don't need to worry during intercourse like taking pills, etc. (laughing)." (RO, 54, Malay)

Menopause was also perceived as a time for religious commitment. Another participant expressed her thoughts as follows:

"We couldn't perform prayer or fasting during menses. Now, we can perform prayer, reciting Quranic verses, and fast comfortably during menopause. At the premenopausal time, we got a certain time to recite the Quran. But, when we reached menopause, every time and every second, we can recite the Quran. No more obstacles compared to those days. I can't say it's an obstacle as it is a natural process of women as God gave us a break from religious duty during menses. We can do religious duty better after menses stopped. It's not proper to call it an obstacle. We are enjoying our lives now." (NS, 55, Malay)

\section{Theme 2: Biopsychosocial changes}

This study's findings validate the changes that women experience during menopause. Some participants reported experiencing physical or somatic symptoms. One of them mentioned:
"I felt pain in my entire body. Sometimes I felt pain over my legs (pointed to both legs). The whole body is in pain, including the muscles. This condition happened since menopause. People said once you reached menopause, you are prone to all the diseases. Back then, I got muscles spasm and had met a doctor. The doctor said it was because of stress. The doctor also asked about my job. Basically, all of these are symptoms of menopause." (RO, 54, Malay)

Furthermore, most experienced psychological and emotional changes that affected their daily lives. For example, one woman asserted:

"In terms of emotions, I do feel terrible. However, there is a time I do feel much better. Previously back then I was not too emotional, and I was able to control it. But now, I realized that I've changed. I'm a bit sensitive. Sometimes, I felt very bad-tempered. Even though it was not so bad, but I could feel that I had changed. Before this, I could accept this as natural, but when I was in menopausal state, I easily felt sensitive, sad, and easily get irritated." (NS, 55, Malay)

\section{Theme 3: Help-seeking behavior}

Help-seeking behavior relates to the behavior that equipped the participants with ways to cope with the symptoms and negative experience of menopause. None of the participants sought professional help. Instead, some used alternative remedies, lifestyle changes, and spiritual practice to overcome these symptoms. Some of the examples they mentioned included the following:

"Nowadays, I feel much better when taking supplements. I consumed various type of supplements every day to boost up my estrogen level and for the skin; for instance primrose oil, calcium tablets as well as vitamin C. I also took 'jamu' (Malay traditional herbs) in a capsule form like 'X'(herb's brand name)." (RO, 54, Malay)

"Mmm... when something irritates me, I keep quiet for a while. By doing this, I could soothe my feelings. I felt much better if I took a rest, zikr (remembrance of God). " (H, 54, Malay)

At the same time, the barriers some of these women reported in terms of seeking treatment involved a lack of knowledge about the availability of treatment and their belief that menopause is a natural course of events for all women. Among the ideas mentioned were these thoughts:

"I don't have any idea about the treatment as well as health services for menopause. I always 
get treatment for my sickness but never heard about it. I never know that menopause has a treatment." (S, 58, Malay)

"I felt not necessary to get treatment. Every woman will experience menopause when the right time comes. I think we need to take milk and supplements when menopause." (A, 57, Malay)

\section{Discussion}

The study findings identified that the women interviewed had different perceptions and underwent varied experiences of menopause. Three major themes were derived from the findings: perception of menopause, biophysical changes, and help-seeking behavior.

\section{Perception of menopause}

The study found that menopause was perceived as an aging process and involved health deterioration. This result is comparable with other studies. ${ }^{29-31}$ The participants believed that reaching menopause is the final road of life, and they accept it wholeheartedly. Nonetheless, aging has been related to a phase that involves deteriorating health and body function, disability, being dependent, and being a burden to others. ${ }^{29}$ Most of the women highlighted changes in facial appearance, skin texture, and body image. They viewed menopause negatively as a time, when growing older, in which all their beauty, youth, and femininity would diminish. ${ }^{33}$ However, this perception also reflects the idea that women tend to embrace menopause as part of a natural process that all women will go through.

Nevertheless, most of the women in our study perceived menopause as a positive experience. They viewed menopause optimistically as a phenomenon that ended the miseries of the reproductive phase, such as menstrual problems and pregnancy, as other studies have reported. ${ }^{31,34,35}$ Bad menstrual experiences, such as cyclical period pain, heavy menses, and fear of pregnancy, finally came to an end upon reaching menopause.

Interestingly, most of the women in this study perceived menopause as a time for them to engage in religious duty, especially among Muslims. This emerging subtheme had not been included in any existing model reviewed. One previous study mentioned that spirituality and religiosity might help women to cope and overcome the menopause life phase better. ${ }^{36}$
The participants generally viewed menopause as bringing an opportunity for them to concentrate more on religious duty that they could not perform during menstruation. Previously, they were bound to their duty towards their family's needs and occupational scope. As they reached menopause, most of them were already retired, allowing them more time to perform religious obligations.

\section{Biopsychosocial changes}

In this study, we found that the participants' most commonly reported symptoms were somatic and psychological. The most common physical symptoms that the women in the study experienced involved musculoskeletal problems, including joint pain, bone-related pain, and body aches, followed by vasomotor symptoms, especially hot flushes and tiredness. This result is comparable with previous studies in other Asian countries ${ }^{37,38}$ and studies done in Malaysia. ${ }^{39,40}$

On the contrary, several studies in Asian and Western countries have reported a higher occurrence of vasomotor and urogenital symptoms than our study participants indicated. ${ }^{41-43}$ The difference in the prevalence of symptoms could be due to estrogen fluctuations that happen mostly in the perimenopausal period as well as psychosocial factors, lifestyle, and culture. Variations in results may also be the result of enormous methodological variation across studies. Additionally, physical symptoms such as tiredness and musculoskeletal problems may coincide with the aging process. ${ }^{44}$

\section{Help-seeking behavior}

Our study findings noted that none of the participants sought treatment for their menopausal symptoms. This outcome could be attributed to a lack of knowledge regarding menopause and its treatment. Huang et al. ${ }^{45}$ reported that about $46 \%$ of Asian women had never read, heard, or seen any information about hormone replacement treatment (HRT). In comparison, European women were more likely to be aware of the availability of HRT. ${ }^{45,46}$

Most of the women in this study preferred natural remedies, supplements, or herbal medication to ease their menopausal symptoms, which is similar to Huang et al.'s findings. ${ }^{45}$ Religious activities, such as performing prayers and reciting the zikr 
(remembrance of God), were also found to be helpful in easing menopausal symptoms. It could be possible that these women did not seek treatment because they considered menopause part of the aging process and a natural occurrence; hence, they tended to embrace it better.

\section{Strengths and limitations of the study}

One strength of this study is that the participants belonged to different socioeconomic backgrounds. Furthermore, the study findings provided a deep understanding of women's perceptions and experiences relating to menopause. However, this study also has some limitations.

In this study, most of the participants were mostly Malay Muslims, limiting the application of these findings to the whole Malaysian population. Health records were not accessed, nor was any investigation undertaken to confirm menopausal status.

\section{Conclusion}

This study's results uncovered a knowledge gap and a lack of support for women impacted by menopause. Muslim women regarded their menopause phase as a time to fully engage in religious duty. Such findings highlight the need for psychosocial intervention and educational programs suited to individuals' cultural and spiritual aspects to support women during this phase of life. Primary healthcare practitioners must be aware of the need for a health promotion program conducted within their healthcare setting to increase women's and families' knowledge of menopause to promote help-seeking behavior and lead to better postmenopausal health for this group of women. ${ }^{7,11}$

\section{Conflict of Interest}

The authors declare no conflict of interest.

\section{Acknowledgment}

We would like to thank all participants who were involved in this study.

\section{Funding}

This research did not receive any specific grant from funding agencies in the public, commercial, or not-for-profit sectors.

\section{How does this paper make a difference to general practice?}

- This study creates and increases awareness that menopausal symptoms are prevalent among Malaysian women and can impact a women's life quality.

- The findings and conclusion stress the importance of educating perimenopausal and menopausal women to seek help in managing their menopausal symptoms.

\section{References}

1. Marahatta RK. Study of menopausal symptoms among peri and postmenopausal women attending NMCTH. Nepal Med Coll J. 2012;14(3):251-255.

2. Goodman NF, Cobin RH, Ginzburg SB, Katz IA, Woode DE. American Association of Clinical Endocrinology medical guidelines for clinical practice for the diagnosis and treatment of menopause. Endocr Pract. 2011;17 (Suppl 6):1-25.
3. Taebi M, Abdolahian S, Ozgoli G, Ebadi A, Kariman N. Strategies to improve menopausal quality of life: a systematic review. J Edu Health Promot. 2018;7(93):1-10.

4. Stanzel KA, Hammarberg K, Fisher J. "Not everybody is an internet person": Barriers for menopause-related health literacy among immigrant women from the Horn of Africa nations. Health Promot J Austr. 2020;00:1-8.
5. Campbell KE, Dennerstein L, Finch S, Szoeke CE. Impact of menopausal status on negative mood and depressive symptoms in a longitudinal sample spanning 20 years. Menopause J North Am Menopause Soc. 2017;24(15):490-496.

6. Currie H, Moger SJ. MenopauseUnderstanding the impact on women and their partners. Post Reprod Health. 2020;25(4):183190. 
7. Hofnie-Hoëbe K, Weightman A, Shatona M, Kelson M. Information sources consulted by women in African countries to manage menopausal symptoms: a systematic review and meta-analysis. Open Journal of Nursing, 2018;(8): 317-338

8. Alshogran OY, Mahmoud FM, Alkhatatbeh MJ. Knowledge and awareness toward menopause and hormone therapy among premenopausal women in Jordan. Climacteric. 2020; (11):1-8.

9. Mustafa GN, Sabir JM. Perception and experience regarding menopause among menopaused women attending teaching hospitals in Erbil city. Glob J Health Sci. 2012;4(3):170-178.

10. Herbert D, Bell RJ, Young K, Brown H, Coles JY, Davis SR. Australian women's understanding of menopause and its consequences: a qualitative study. Climacteric. 2020; 23 (6):622-628.

11. Stanzel KA, Hammarberg K, Fisher J. Experiences of menopause, self-management strategies for menopausal symptoms and perceptions of health care among immigrant women: a systematic review. Climacteric. 2018;21(2):101-110

12. Joseph L, Varghese AP. Prevalence of menopausal symptoms and perceptions about menopause among postmenopausal women attending Gynaecology OPD at GMC Idukki, India. Int J Reprod Contracept Obs Gynecol. 2017;6(2):413-441.

13. Ghazanfarpour M, Khadivzadeh T, Latifnejad Roudsari R, Mehdi Hazavehei SM. Obstacles to the discussion of sexual problems in menopausal women: a qualitative study of healthcare providers. J Obstet Gynaecol. 2017;37(5):660-666.

14. Faccio E, Solarino M, Vitelli R, and Cipolletta S. Normalisation versus medicalisation of sexual disturbances during menopause: a qualitative research in the Italian context. Sexuality \& Culture. 2018; 22(2), 445-461.

15. Abdullah B, Moize B, Ismail BA, Zamri M, Mohd Nasir NF. Prevalence of menopausal symptoms, its effect on quality of life among Malaysian women and their treatment seeking behaviour. Med J Malaysia. 2017;72(2):9499.
16. Hassan SS, Thiruchelvam K, Ahmed SI, Clavarino AM, Mamun AA, Kairuz T. Psychological health and menopause-specific quality of life of Malaysian women with type 2 diabetes. Asian J Psychiatr. 2016; 23:56-63.

17. Diah NM. The knowledge of menopause and its treatment among Orang Asli in Gombak. Int Res J Soc Sci. 2013;2(8):1-4.

18. Diah NM. Exploring a sensitive issue: menopause experience among urban Malay women in Malaysia. 2007 [proceeding]

19. Quiroga A, Larroy C, Gonzalez-Castro P. Climacteric symptoms and their relation to feminine self-concept. Climacteric 2017;20(3):274-279.

20. Schneider HPG, Birkhäuser M. Quality of life in climacteric women. Climacteric 2017;20 (3):187-194.

21. Leventhal H, Leventhal EA, Contrada RJ. Self-regulation, health, and behavior: a perceptual cognitive approach. Psychology \& Health 1998;13:717-733.

22. Pimenta F, Ramos MM, Silva CC, Costa PA, Maroco J, Leal I. Self-regulation model applied to menopause: a mixed-methods study. Climacteric.2020 23(1), 84-92.

23. Meriam S B, Tisdell EJ. Designing your study and selecting a sample. In Qualitative Research-A Guide to Design and Implementation 2016 (pp. 95-98).

24. Palinkas LA, Horwitz SM, Green CA, Wisdom JP, Duan N, Hoagwood K. Purposeful sampling for qualitative data collection and analysis in mixed method implementation research. Adm Policy Ment Health.2015;42(5):533-544.

25. Powell RA, Single HM. Methodology matters-v focus groups. International Journal for Quality in Health Care, 1996.8(5), 499-504.

26. Hakimi S, Simbar M, Tehrani F. Perceived concerns of Azeri menopausal women in Iran. Iranian Red Crescent Medical Journal. 2014; 16(5), 1-7.
27. Dworkin SL. Sample size policy for qualitative studies using in-depth interviews. Arch Sex Behav. 2012;41(6):1319-1320.

28. Gill P, Stewart K, Treasure E, Chadwick B. Methods of data collection in qualitative research: interviews and focus groups. Br Dent J. 2008; 204(6), 291-295.

29. Sakdiah MH, Sulaiman H, Zulkefli NA. Views on ageing: a qualitative study among malay middle-aged women in urban setting in Malaysia. Int J Heal Sci Res. 2015;5(April):248-256.

30. Wong LP, Nur Liyana AH. A survey of knowledge and perceptions of menopause among young to middle-aged women in federal territory, Kuala Lumpur, Malaysia. J Univ Malaya Med Cent. 2007;10(2):22-30.

31. Mahadeen AI, Halabi JO, Callister LC. Menopause: a qualitative study of Jordanian women's perceptions. Int Nurs Rev. 2008;55:427-33.

32. Braun V, Clarke V. Using thematic analysis in psychology. Qualitative Research in Psychology, 2006 3(2), 77-101.

33. Anderson D, Posner N. Relationship between psychosocial factors and health behaviours for women experiencing menopause. Int J Nurs Pract. 2002;8:265-273.

34. Brown L, Brown V, Judd F, Bryant C. It's not as bad as you think: menopausal representations are more positive in postmenopausal women. J Psychosom Obstet Gynecol. 2018;39(4):281-288.

35. Im E-O, Lee SH, Chee W. "Being conditioned, yet becoming strong": Asian American Women in Menopausal Transition. J Transcult Nurs. 2011;22(3):290-299.

36. Strezova A, O’Neill S, O'Callaghan C, Perry A, Liu J, Eden J. Cultural issues in menopause: an exploratory qualitative study of Macedonian women in Australia. Menopause. 2017;24(3):308-315.

37. Shea JL. Chinese women's symptoms: relation to menopause, age and related attitudes. Climacteric. 2006;9(1):30-9. 
38. Li L, Wu J, Pu D, Zhao Y, Wan C, Sun L, et al. Factors associated with the age of natural menopause and menopausal symptoms in Chinese women. Maturitas. 2012;73(4):354360 .

39. Dhillon HK, Singh HJ, Shuib R, Hamid AM, Mohd Zaki Nik Mohamad N. Prevalence of menopausal symptoms in women in Kelantan, Malaysia. Maturitas. 2006;54(3):213-221.

40. Syed Alwi SA, Lee PY, Awi I, Malik PS, Md Haizal MN. The menopausal experience among indigenous women of Sarawak, Malaysia. Climacteric. 2009;12(6):548-556.
41. Chuni N, Sreeramareddy CT. Frequency of symptoms, determinants of severe symptoms, validity of and cut-off score for Menopause Rating Scale (MRS) as a screening tool: a cross-sectional survey among midlife Nepalese women. BMC Women's Health. 2011;11(30):1-9.

42. Yanikkerem E, Koltan SO, Tamay AG, Dikayak S. Relationship between women's attitude towards menopause and quality of life. Climacteric. 2012;(15):552-562.

43. Ali S, Tarin AU, Bhatti R. Average age of menopause and its climacteric symptoms in women of Multan city. Pakistan Journal Medical Health Sciences. 2015;9(1):262-4.
44. Rahman SASA, Zainudin SR, Mun VLK. Assessment of menopausal symptoms using modified Menopause Rating Scale (MRS) among middle age women in Kuching, Sarawak, Malaysia. Asia Pac Fam Med. 2010;9(1):5.

45. Huang KE, Xu L, I NN, Jaisamrarn U. The Asian Menopause Survey: knowledge, perceptions, hormone treatment and sexual function. Maturitas. 2010;65(3):276-283.

46. Genazzani AR, Schneider HP, Panay N, Nijland EA. The European Menopause Survey 2005: women's perceptions on the menopause and postmenopausal hormone therapy. Gynecol Endocrinol. 2006;22(7):369-375. 